\title{
Study on the LED Lights Intelligent Control System Based on ZigBee Technology
}

\author{
Jie Fang, Xiang Yang \\ College of Mechanical and Electronic Engineering, \\ West Anhui University, \\ Liu’an,Anhui 237012,China \\ 63640193@qq.com
}

\begin{abstract}
This paper introduces a home networking solution of wireless intelligent control lighting system with Zig-Bee to overcome the shortage of the traditional intelligent light control system. Software and hardware design of networking are also made.Then based on the main control chip CC2430, the coordinator, routers and terminals in networking are developed to realize wireless intelligent control LED with ZigBee.
\end{abstract}

Keywords-intelligent lighting system; RF communication; ZigBee; CC2430;energy saving

\section{INTRODUCTION}

Most of the traditional intelligent lighting systems such as Lon Works, Cebus, X-10, CAN bus and Ethernet[1-2] are installed with cable.These methods have the advantages and disadvantages respectively ,X-10 system has the advantages of low price and easy disassemble ,which has decent market acceptance, but not very good performance. Cebus and Lon Works have a better performance, but the price are expensive and require professional auxiliary installation. RS485 and CAN bus applied in industrial, civilian popularityare not high . Ethernet transmission rate is high, but the space of wiring required is relatively large, not good application in a limited area of the houses[3-5].

In this paper, we propose an adaptive control scheme of LED lights based on ZigBee, and design the software and hardware required by the network. The system uses CC2430 chip as the main control chip, the passive infrared sensor and a photosensitive sensor are used to detect the change of the environment ,then the network coordinator, router and terminal equipment are designed to realize wireless intelligent control of LED lights.

\section{II.THE DESIGNING METHOD}

The program flow chart of the control sending nodes are shown in figure 1, the system is consists of two parts which contains the control sending and receiving controlled nodes.Controlling the photosensitive sensor and PIR sensor in the sending node, when the photosensitive sensor and PIR sensor output high electricity at ordinary times, that's to say to send wireless signal when in the situation of having someone but no light, the receiving controlled side will receive signal and light LED up.In the rest of the case (having light but

\author{
Ping Yu, Zhi-yuan Weng, Min Kong \\ Intelligent Lighting and Display Technology Center \\ West Anhui University \\ Liu’an, Anhui237012,China
}

nobody , no light and nobody, having light and someone ),it doesn't send signals or light LED up, so as to realize adaptive control.

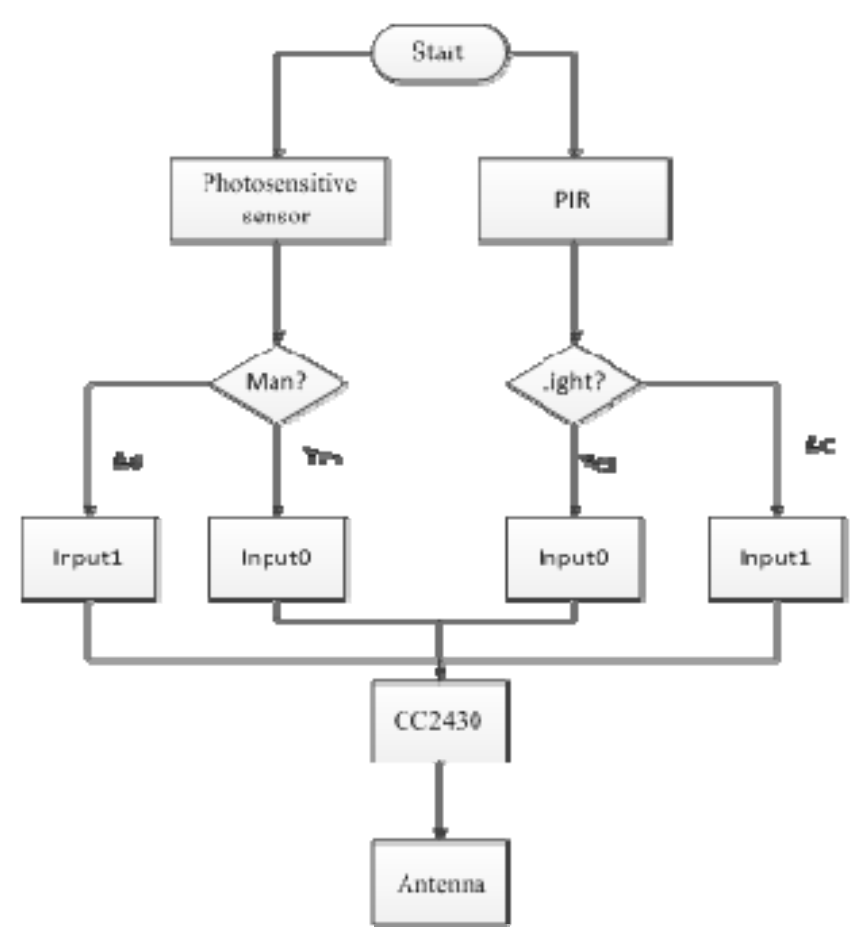

Figure 1. The program flow chart of the control sending nodes

\section{DESIGNING THE NODE CIRCUITS}

The transmitting terminal and the receiving terminal in the system is composed of core board and functional floorboard.Core board contains CC2430 module, antenna module and power module, which realizing the function of sending and receiving data. the schematic diagram is shown in figure 2 . 


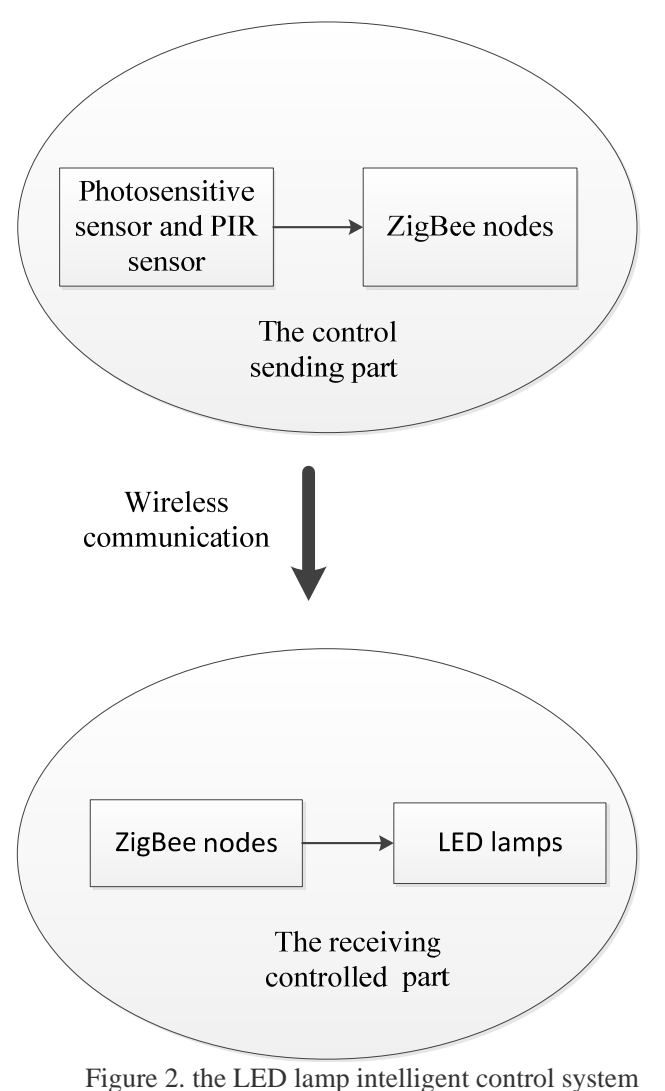

The control module is composed of the photosensitive sensor and PIR sensor and a module, another module as the receiving node controls LED lights.The sensors control the detecting signal send to receiving module by the antenna, the receiving module have a corresponding control to LED according to the received signal, and to realize the wireless control and adaptive control of LED lights.

Network controls node circuit diagram as shown in figure 3.The serial port part has completed the conversion between RS232 and TTL level, which used for communication between computer and the coordinator nodes of ZigBee wireless network.Among which photoelectric diode D1 is used for power light,similarly,D2 and D3 for network demonstration pilot light.Terminal receiving node is made up of CC2430 typical circuit plus some necessary circuit.in which the resisters packs are $470 \mathrm{Ohm}$ current limiting resistor, DuPont line as the electric wires, the power source is depended on $3.3 \mathrm{~V}$ power supply module,and the diode adopts common-anode method, the cathode of diode connected to the I/O module by dupont line, LED will light when the I/O outputs low levels.

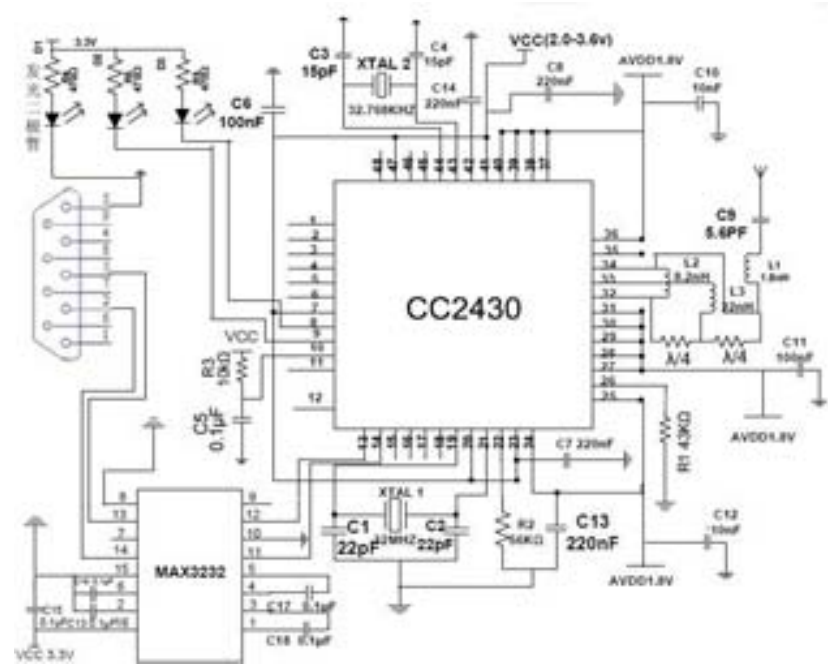

Figure 3. the control node circuit diagram

\section{IV.EXPERIMENTS}

\section{A.The LED Adaptive On-off Control Experiment}

At the beginning of the network experiment, Z-Stack will initialize first and then the operating system try to query, the photosensitive sensor and PIR sensor will compile, link, and loading to the sensor node program when they are inserted into the corresponding place of the control module.Go up to two modules to electricity, press the reset button.To facilitate the PIR sensor detection,whether there is someone in the test with the "hand" to replace,The Light source, which provided by a lamp is convenient for photoelectric sensors detecting .the system is made up four kinds of working modes: light and someone ,having light but nobody, no light and nobody, having someone but no light, among four modes the green LED indicator will light only in the mode of having someone but no light[6-8].

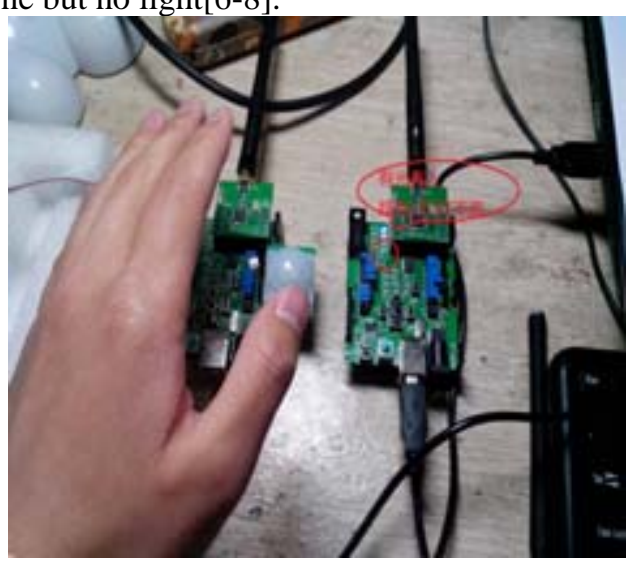

(a) having light and someone 


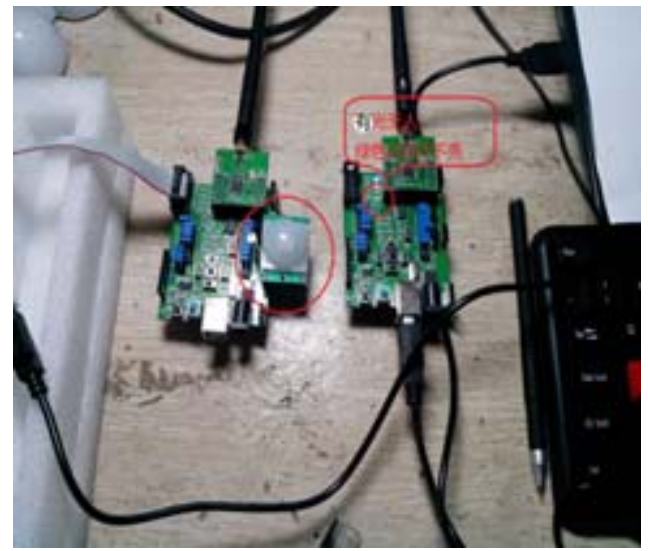

(b) having light but nobody

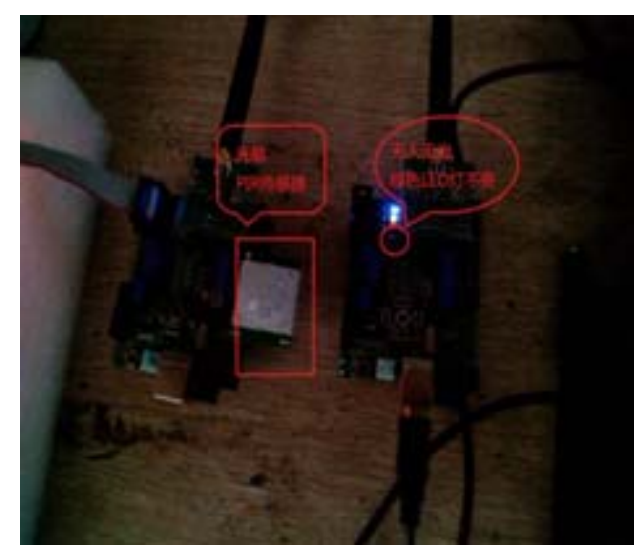

(c) no light and nobody

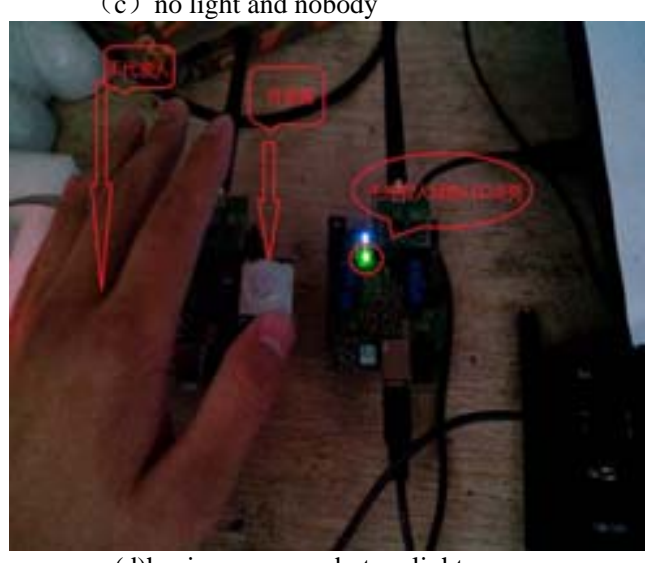

(d)having someone but no light

Figure 4.The LED adaptive on-off control experiment

\section{B.Keys Wireless Control Heart LED Lights Experiment}

Preparing the heart LED lights that you want to use and other tools before opening the IAR Embedded Workbench IDE software. Adding the project to the software later, selecting coordinator and end pointer choice to debug the two modules respectively, after finishing compiling and assembly linking into object code, then fulfilling the object code loading. Next, supplying power to two module by using dry battery independently, pressing the reset button again, waiting for establishing network and then we can start experiment.

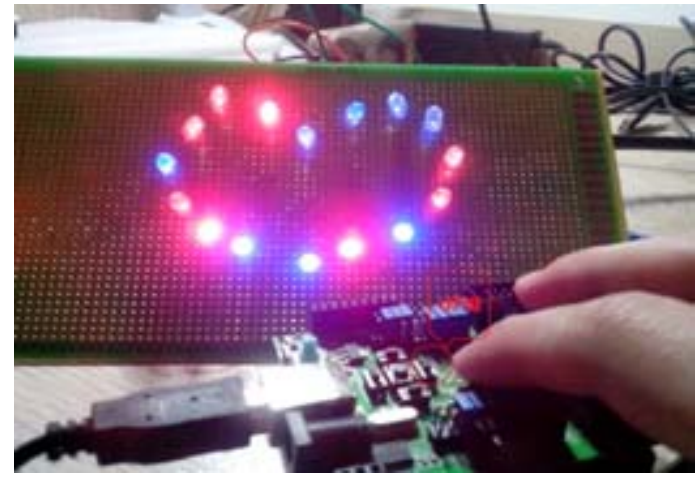

Figure 6 The experiment of the button control LED lamp

Moreover, the experiment can realize various dynamic working modes of the LED lighting and flashing alternately in wireless controlling through different program codes.

\section{V .SUMMARY}

This paper uses ZigBee technology to realize the wireless intelligent control to LED lights, the technology possesses a variety of advantages such as high degree intelligent, convenient networking, and diverse control modes.This work has practical significance, it has provided the basis and direction for research work, if we substitute the STM single chip computer and 2.4G radio frequency chip for ZigBee nodes, making a smaller circuit board and packaging in the LED base part as the terminal equipment, which can greatly reduce cost and enhance the market competition ability of LED intelligent lights.

\section{ACKNOWLEDGMENT}

This work is supported by NFSC(61302179, 61303209), and key science foundation for outstanding yong talent of Anhui (2013SQRL072ZD),and Anhui provincial natural science foundation(1408085MA20, 1301023004), and the natural science research project of West Anhui University (0041014006).

\section{REFERENCES}

[1] Qi,Dai.Design of Wireless Streetlight Control System Based on Zigbee[D].Xidian University,2012

[2] Jian Yu.The Research of Intelligent Lighting Control System of LED Based on ZigBee Wireless Sensor Network Technology[D].Zhejiang University of Technology,2012

[3] Hai -tao Wang ,Zhao-you Zhu.Econanica1 Street LED Lamp Contro1 Systan Based on ZigBee[J].Journal of east china institute technology.2009(12): 394-396

[4] Jing Chen,Jing-dong Wu. Analysis and Application of Wireless Sensor Network Based on ZigBee Protocol[J].Industrial Control Computer.2011(11):30-31

[5] Wheeler A. Commercial applications of wireless sensor networks using ZigBee[J]. IEEE communications magazine, 2007,45(4):70-77

[6] Wu-bin Zhou . Research on ZigBee wireless networking technology[D].Central South University ,2009

[7] Akyldiz IF, Su W, Sankarasubramanian Y, Cayirci E. Wireless sensor networks: A Survey [J].Computer Networks, Mar., 2012, 38(04): 393-422.

[8] Ye Xiaojing, Huang Junwei. A framework for Cloud-based Smart Home[C]. 2011 International Conference on Computer Science and Network Technology, 2011(2):894-897. 